\title{
AlphaSpace 2.0: Representing Concave Biomolecular Surfaces using Beta-Clusters
}

Joseph Katigbak, ${ }^{1}$ Haotian Li ${ }^{1}$, David Rooklin ${ }^{1}$, and Yingkai Zhang ${ }^{1,2 *}$

1) Department of Chemistry, New York University, New York, NY 10003 USA

2) NYU-ECNU Center for Computational Chemistry at NYU Shanghai, Shanghai 200062, China

\section{Supporting Information}

Figure S1 and Figure S2 


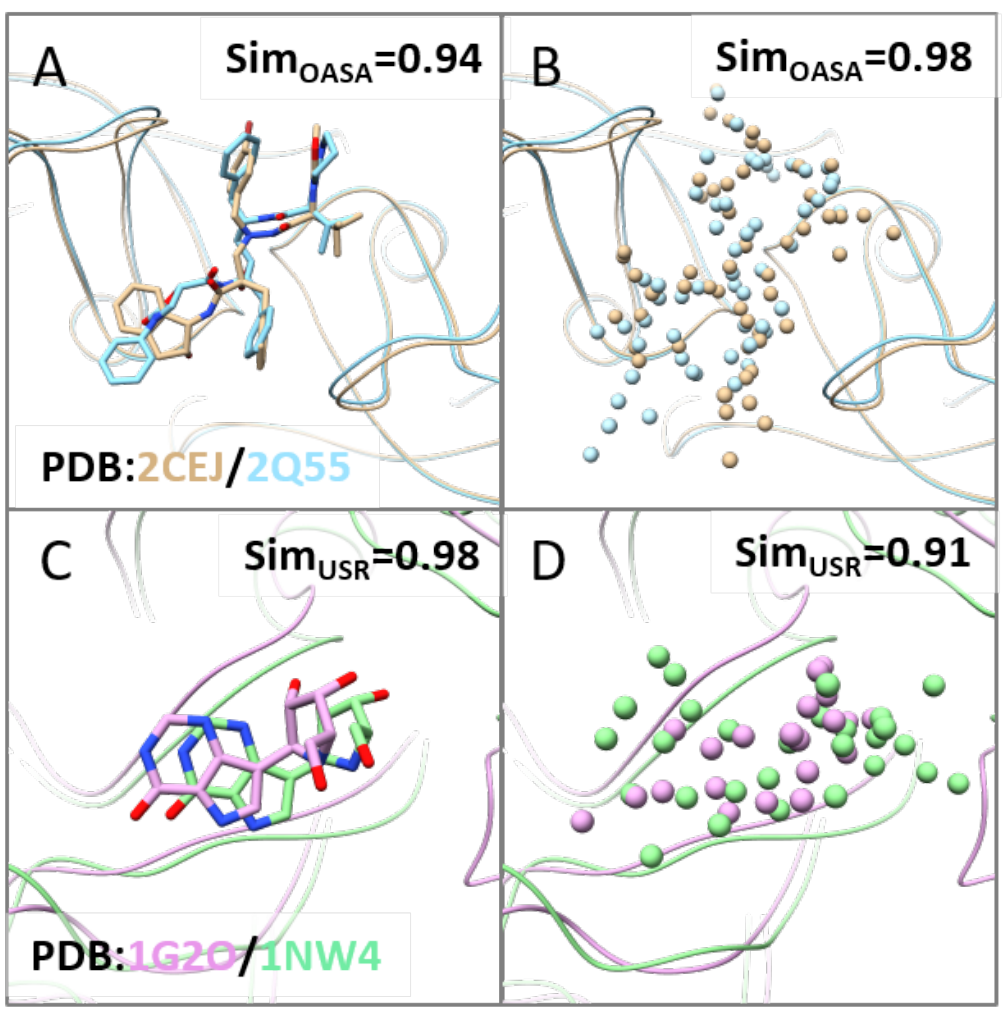

Figure S1 Representative ligand and beta cluster pairs that have high similarity $(>0.90)$ for the Occluded ASA and USR. The ligand beta cluster pairs in (A, B) correspond to two variants of HIV-1 Protease and were classified as highly similar based on the Occluded ASA similarity. (C, D) correspond to purine nucleoside phosphorylase from two different species with a high pairwise USR similarity. 

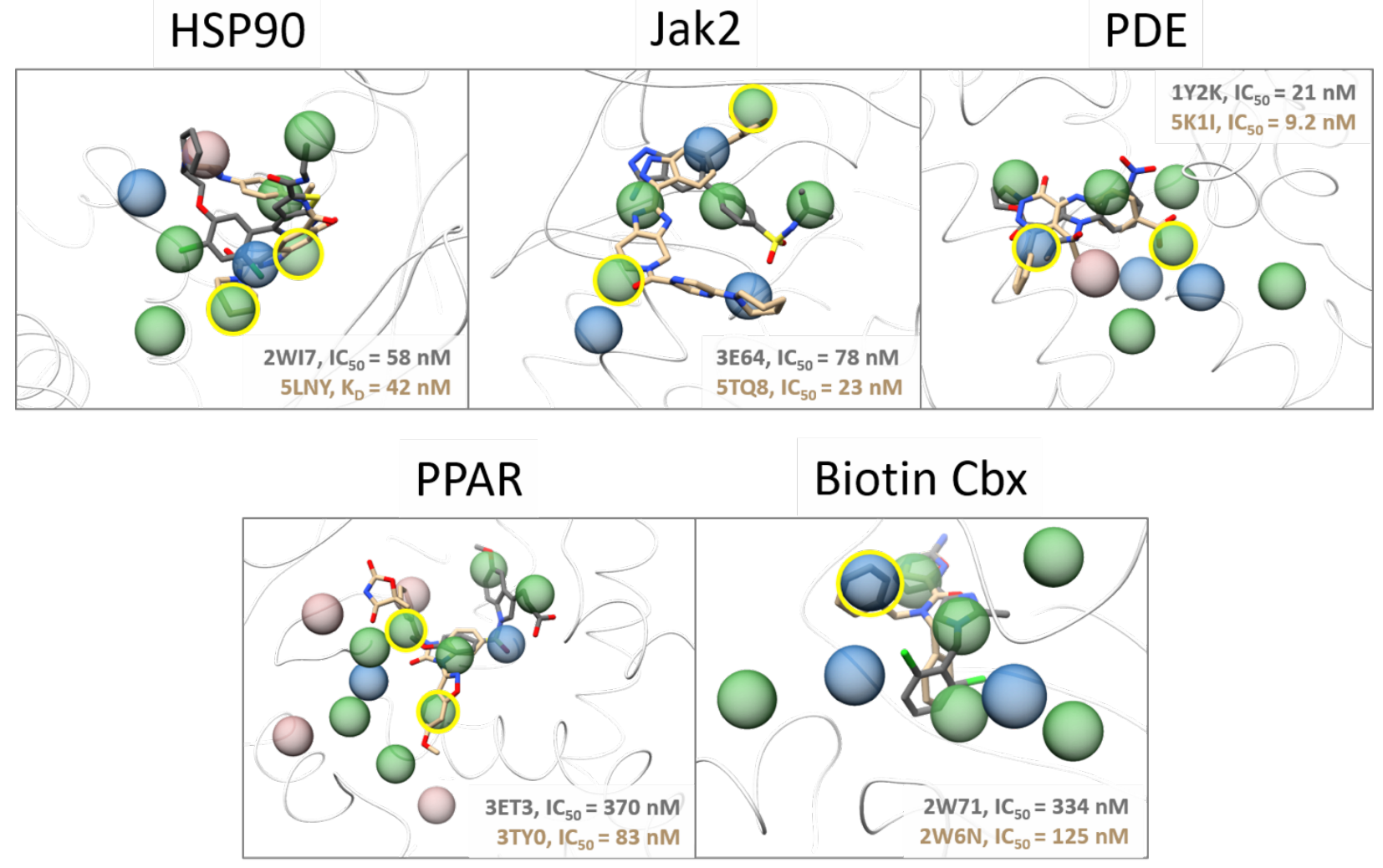

Figure S2 Selected systems from the Ichihara set where multiple, high and mid-scoring pockets where identified for the binding site communities that had limited overlap with the original lead molecule. Lead molecules from the Ichihara set are shown and colored dark grey while ligands from the PDBBind that targeted these alternative pockets are shown in tan. High (BScores $<-2.5$ $\mathrm{kcal} / \mathrm{mol})$, $\mathrm{mid}(-2.5 \mathrm{kcal} / \mathrm{mol}>=\mathrm{BScores}<-1.5 \mathrm{kcal} / \mathrm{mol})$, and low (BScores $>=-1.5 \mathrm{kcal} / \mathrm{mol})$ scoring pockets are colored green, blue, and rosy brown respectively. Pockets that overlap extensively with the novel ligands are highlighted in gold. 